\title{
On the Bounds of the First Reformulated Zagreb Index
}

\author{
T. Mansour ${ }^{1}$, M. A. Rostami ${ }^{2}$, E. Suresh ${ }^{3, *}$, G. B. A. Xavier ${ }^{4}$ \\ ${ }^{1}$ Department of Mathematics, University of Haifa, 3498838 Haifa, Israel \\ ${ }^{2}$ Institute for Computer Science, Friedrich Schiller University Jena, Germany \\ ${ }^{3}$ Department of Mathematics, Velammal Engineering College, Surapet, Chennai-66, Tamil Nadu, India \\ ${ }^{4}$ Department of Mathematics, Sacred Heart College, Tirupattur-635601, Tamil Nadu, India \\ *Corresponding author: sureshkako@gmail.com
}

Received December 11, 2015; Revised February 02, 2016; Accepted February 10, 2016

\begin{abstract}
The edge version of traditional first Zagreb index is known as first reformulated Zagreb index. In this paper, we analyze and compare various lower and upper bounds for the first reformulated Zagreb index and we propose new lower and upper bounds which are stronger than the existing and recent results [Appl. Math. Comp. 273 (2016) 16-20]. In addition, we prove that our bounds are superior in comparison with the other existing bounds.
\end{abstract}

Keywords: Zagreb indices, first reformulated Zagreb index, forgotten topological index

Cite This Article: T. Mansour, M. A. Rostami, E. Suresh, and G. B. A. Xavier, "On the Bounds of the First Reformulated Zagreb Index.” Turkish Journal of Analysis and Number Theory, vol. 4, no. 1 (2016): 8-15. doi: 10.12691/tjant-4-1-2.

\section{Introduction}

Throughout this paper, we consider finite connected undirected simple graphs. Let $G$ be such a graph with $n$ vertices and $m$ edges. The degree of a vertex $u$ is denoted by $d(u)$. Especially, $\Delta=\Delta(G)$ and $\delta=\delta(G)$ are called as maximum and minimum degree of $G$, espectively. The degree of an edge is denoted by $d(e)$ in $G$, which is defined by $d(e)=d(u)+d(v)-2$ for $e=u v \in E(G)$. Let $\bar{G}$ denotes the complement of $G$, with the same vertex set such that two vertices $u$ and $v$ are adjacent in $\bar{G}$ if and only if they are not adjacent in $G$.

The first and second Zagreb indices are defined as (for instance, see [9] and references cited therein)

$$
\begin{aligned}
& M_{1}^{2}(G)=\sum_{v \in V(G)} d(v)^{2} \\
& \text { and } M_{2}^{1}(G)=\sum_{u v \in E(G)} d(u) d(v) .
\end{aligned}
$$

Numerous papers were recorded in the literature regarding the mathematical and chemical properties on Zagreb indices and the surveys on Zagreb indices. For the recent outcomes on Zagreb indices, see [5] and references cited therein. In 2005, Li and Zheng [13] introduced the the generalized version of the first Zagreb index. For $\alpha \in \mathbb{R}$ and $G$ be any graph, it satisfies

$$
M_{1}^{\alpha+1}(G)=\sum_{v \in V(G)} d(v)^{\alpha+1}=\sum_{u v \in E(G)}\left[d(u)^{\alpha}+d(v)^{\alpha}\right] .
$$

In 2015, Fortula and Gutman [8] re-introduced the forgotten topological index:

$$
F(G)=M_{1}^{3}(G)=\sum_{v \in V(G)} d(v)^{3} .
$$

Mathematical properties of the forgotten topological index is seen in $[4,8,11,19]$. Miličević, Nikolić and Trinajstić [15] reformulated the first Zagreb index in terms of edge-degree instead of vertex-degree:

$$
E M_{1}^{2}(G)=\sum_{e \in E(G)} d(e)^{2} .
$$

The first reformulated Zagreb index is simply the Zagreb index of its line graph $L(G)$. The use of these descriptors in QSPR study was also discussed in their report [15]. In [6,11,12,17,20], various properties, relations and bounds are also explored.

The inverse degree was first appeared through conjectures of the computer program Graffiti [7] denote by $I D(G)$; with no isolated vertices defined by

$$
I D(G)=\sum_{v \in V(G)} \frac{1}{d(v)} .
$$

The inverse degree is a special case formula at $\alpha=-1$ in (1.1). For the recent results of the inverse degree, see $[3,18]$. In analogy, the inverse edge degree of a graph $G$ with no isolated edges is defined by

$$
\operatorname{IED}(G)=\sum_{e \in E(G)} \frac{1}{d(e)} .
$$

This paper is organized as follows. In Section 2, we give some preliminaries and basic definitions that will be used throughout the paper. In Sections 3 and 4, we present lower and upper bounds on the first reformulated Zagreb index $E M_{1}^{2}(G)$ in terms of $n, m, \Delta, \delta, I D(G), \operatorname{IED}(G)$, $M_{1}^{2}(G)$ and $M_{2}^{1}(G)$ respectively. Furthermore, (Refer Table 3- Table 6) we showed that our bounds have the smallest deviation from the first reformulated Zagreb 
index $E M_{1}^{2}(G)$ and superior than the existing bounds in the literature.

\section{Preliminaries}

As usual $P_{n}, K_{1, n-1}$ and $C_{n}$ denote a path, star and a cycle of order $n$, respectively. Let $G$ and $H$ be graphs. We denote the number of distinct subgraphs of the graph $G$ which are isomorphic to $H$ by $\sigma_{G}(H)$. In specific, $G$ has $\sigma_{G}\left(P_{1}\right)$ vertices, $\sigma_{G}\left(P_{2}\right)$ edges and $\sigma_{G}\left(C_{3}\right)$ triangles. Let $\alpha$ and $\beta$ be positive integers, then the double star $D_{\alpha, \beta}$ is a tree on $\alpha+\beta+2$ vertices obtained from the path $P_{2}$, by attaching $\alpha$ pendent vertices to its one vertex, and $\beta$ pendent vertices to its other vertex.

GraphTea [1] is a software tool for graph problems with a focus on extracting information and visualization. It offers powerful ways to query or directly interact with properties of a particular instance of a graph problem. It is specially designed for the computational works on the topological indices.

To analyse and compare our results, we need to recall few classes of graphs:

- The wheel $W_{n}$ is join of the graphs $C_{n-1}$ and $K_{1}$.

- The crown $C r_{n}$ is obtained from the cycle $C_{n}$ by adjoining a pendant edge at each vertex of the cycle.

- The helm $H_{n}$ is obtained from the wheel graph $W_{n-1}$ by adjoining a pendant edge at each vertex of the cycle.

- The flower $F l_{n}$ is obtained from the helm $H_{n}$ by joining each pendent vertex to the central vertex of the helm.

- A graph $G$ is called bidegreed if its vertex degree is either $\Delta$ or $\delta$ with $\Delta>\delta \geq 1$.

In 2010, Zhou and Trinajstić [20] obtained the following identity.

Proposition 2.1. Let $G$ be a simple graph with $n$ vertices and $m$ edges. Then

$$
E M_{1}^{2}(G)=M_{1}^{3}(G)+2 M_{2}^{1}(G)-4 M_{1}^{2}(G)+4 m .(2.1)
$$

Using the results from [4] without exaggeration, we obtain the following corollary for $\operatorname{EM}_{1}^{2}(G)$ in terms of its subgraphs of $G$.

Corollary 2.2. Let $G$ be a simple graph with $n$ vertices and $m$ edges. Then

$$
E M_{1}^{2}(G)=6 \sigma_{G}\left(K_{1,3}\right)+2 \sigma_{G}\left(P_{4}\right)+2 \sigma_{G}\left(P_{3}\right)+6 \sigma_{G}\left(P_{3}\right) .
$$

\section{Lower Bounds on the First Reformulated Zagreb Index}

In 2012, Ilić and Zhou [11] proposed the following lower bound for $E M_{1}^{2}(G)$.

Lemma 3.1. Let $G$ be a simple graph with $n$ vertices and medges. Then

$$
E M_{1}^{2}(G) \geq \frac{2 m}{n} M_{1}^{2}(G)+2 M_{2}^{1}(G)-4 M_{1}^{2}(G)+4 m(3.1)
$$

with equality if and only if $G$ is regular.

Very recently in 2016, Milovanović, Dolićanin, Glogić [16] obtained the new lower bounds for $\operatorname{EM}_{1}^{2}(G)$ in terms of $M_{1}^{2}(G)$ and $M_{2}^{1}(G)$.

Lemma 3.2. Let $G$ be a simple graph with $n(n \geq 2)$ vertices and $m$ edges. Then

$$
\begin{gathered}
E M_{1}^{2}(G) \geq \frac{M_{1}^{2}(G)^{2}}{2 m}+2 M_{2}^{1}(G)-4 M_{1}^{2}(G)+4 m(3.2) \\
E M_{1}^{2}(G) \geq 4\left(M_{2}^{1}(G)-M_{1}^{2}(G)+m\right)
\end{gathered}
$$

equality holds if and only if $G$ is a regular.

Remark 3.3. In 2015, Fortula and Gutman [8] have given the following lower bounds for $M_{1}^{3}(G)$. Let $G$ be a graph with $m$ edges. Then

$$
\begin{gathered}
M_{1}^{3}(G) \geq \frac{\left(M_{1}^{2}(G)\right)^{2}}{2 m} \\
M_{1}^{3}(G) \geq \frac{\left(M_{1}^{2}(G)\right)^{2}}{2 m}-2 M_{2}^{1}(G) .
\end{gathered}
$$

Note that, the equality of (3.4) holds if and only if $G$ is regular. It is easy to see that, the inequality (3.2) can be obtained from (2.1) by using (3.4).

In analogy, one can propose a lower bound for $E M_{1}^{2}(G)$ using (3.5). But in 2012, De [6] obtained the following inequality.

Lemma 3.4. Let $G$ be a simple graph with $n(n \geq 2)$ vertices and $m$ edges. Then

$$
E M_{1}^{2}(G) \geq \frac{\left(M_{1}^{2}(G)\right)^{2}}{m}-4 M_{1}^{2}(G)+4 m
$$

equality holds if and only if $G$ is a regular.

In 2010, Zhou and Trinajstić [19] proposed the following inequality in the context of general sum connectivity.

Lemma 3.5. Let $G$ be a graph with $m \geq 1$ edges. If $0<\alpha<1$, then $\chi^{\alpha}(G) \leq M_{1}^{2}(G)^{\alpha} m^{1-\alpha}$, and if $\alpha<0$ or $\alpha>1$, then $\chi^{\alpha}(G) \geq M_{1}^{2}(G)^{\alpha} m^{1-\alpha}$, and either equality holds if and only if $d(u)+d(v)$ is a constant for any edge $u v$.

Note that, for $\alpha=2$ in Lemma 3.5 turns (3.5) as its special case. Also from Lemma 3.5, it is clear that the equality of (3.6) holds if and only if $d(u)+d(v)$ is a constant for any edge $u v$ : In addition, from [8], it is easy to see that (3.2) and (3.6) are incomparable.

Now, we are ready to present our lower bounds on $E M_{1}^{2}(G)$ in terms of $n, m, \Delta, \delta, I D(G), M_{1}^{2}(G)$ and $M_{2}^{1}(G)$.

Theorem 3.6. Let $G$ be a simple graph with $n$ vertices and $m$ edges, then 


$$
\begin{aligned}
E M_{1}^{2}(G) \geq & \frac{M_{1}^{2}(G)^{2}}{2 m}+\frac{1}{2 m}\left(2 m I D(G)-n^{2}\right) \\
& +2 M_{2}^{1}(G)-4 M_{1}^{2}(G)+4 m
\end{aligned}
$$

equality holds if and only if $G$ is regular.

Proof. Consider $w_{1}, w_{2}, \ldots, w_{k}$ be the non-negative weights, then we have the weighted version of Cauchy-Schwartz inequality

$$
\sum_{i=1}^{k} w_{i} a_{i}^{2} \sum_{i=1}^{k} w_{i} b_{i}^{2} \geq\left(\sum_{i=1}^{k} w_{i} a_{i} b_{i}\right)^{2}
$$

Since $w_{i}$ is non-negative, we assume that $w_{i}=m_{i}-n_{i}$ such that $m_{i} \geq n_{i} \geq 0$. Thus

$$
\begin{aligned}
& \sum_{i=1}^{k} m_{i} a_{i}^{2} \sum_{i=1}^{k} m_{i} b_{i}^{2}-\left(\sum_{i=1}^{k} m_{i} a_{i} b_{i}\right)^{2} \\
& \geq \sum_{i=1}^{k} n_{i} a_{i}^{2} \sum_{i=1}^{k} n_{i} b_{i}^{2}-\left(\sum_{i=1}^{k} n_{i} a_{i} b_{i}\right)^{2} \geq 0
\end{aligned}
$$

Set $m_{i}=d\left(v_{i}\right), n_{i}=\frac{1}{d\left(v_{i}\right)}, a_{i}=d\left(v_{i}\right)$ and $b_{i}=1$, for all $i=1,2, \ldots, n$ in the above, we get

$$
\begin{aligned}
& \sum_{i=1}^{n} d\left(v_{i}\right)^{3} \sum_{i=1}^{n} d\left(v_{i}\right)-\left(\sum_{i=1}^{n} d\left(v_{i}\right)^{2}\right)^{2} \\
& \geq \sum_{i=1}^{n} d\left(v_{i}\right) \sum_{i=1}^{n} \frac{1}{d\left(v_{i}\right)}-\left(\sum_{i=1}^{n} 1\right)^{2} .
\end{aligned}
$$

The inequality (3.7) is obtained from the above inequality and equality (2.1) and the equality holds if and only if $G$ is regular.

Theorem 3.7. Let $G$ be a simple graph with $n$ vertices and $m$ edges, then

$$
\begin{aligned}
E M_{1}^{2}(G) \geq & \frac{M_{1}^{2}(G)^{2}}{2 m}+\frac{1}{2 m}\left(n M_{1}^{2}(G)-4 m^{2}\right) \\
& +2 M_{2}^{1}(G)-4 M_{1}^{2}(G)+4 m
\end{aligned}
$$

equality holds if and only if $G$ is regular.

Proof. The proof follows from the same terminology of Theorem 3.6 by choosing $m_{i}=d\left(v_{i}\right), n_{i}=1, a_{i}=d\left(v_{i}\right)$ and $b_{i}=1$, for all $i=1,2, \ldots, n$.

Remark 3.8. For every simple graph $G$, the lower bound in (3.10) is always better than the lower bound in (3.7). For this, we have to show that

$$
n M_{1}^{2}(G)-4 m^{2} \geq 2 m I D(G)-n^{2} \geq 0
$$

By fixing $a_{i}=d\left(v_{i}\right), b_{i}=1, m_{i}=1$ and $n_{i}=d\left(v_{i}\right)^{-1}$ in (3.9), we achieve our required claim and the equality holds if and only if $G$ is regular. Therefore inequality (3.10) is stronger than (3.7) and (3.7) is stronger than (3.2).

Remark 3.9. On the other hand, the lower bounds in (3.6) and (3.10) are incomparable. For Helm $H_{n}(n \geq 4)$ the lower bound in (3.10) is better than (3.6) and for the Flower $F_{n}(n \geq 4)$ the lower bound in (3.6) is better than (3.10). In addition for the Crown $\mathrm{Cr}_{n}$ both the lower bounds coincides together, other than the equality case.

Next, we need to improve the lower bound (3.6). For which we need the following inequality which relates the first Zagreb index, second Zagreb index and the forgotten topological index.

Theorem 3.10. Let $G$ be a simple graph with no isolated edges, then

$$
\begin{aligned}
M_{1}^{3}(G) \geq & \frac{M_{1}^{2}(G)^{2}}{m}+\frac{1}{m}\left[M_{1}^{2}(G)-2 m\right] \operatorname{IED}(G) \\
& -2 M_{2}^{1}(G)-m
\end{aligned}
$$

equality holds if and only if $d(u)+d(v)$ is a constant for any edge uv.

Proof. Considering the weighted version of CauchySchwartz inequality from (3.8) with the assumption $w_{i}=x_{i}-y_{i}$ such that $x_{i} \geq y_{i} \geq 0$ and by setting $x_{i}=1$, $y_{i}=\frac{1}{d\left(e_{i}\right)}, a_{i}=d\left(e_{i}\right)$ and $b_{i}=1$, for all $i=1,2, \ldots, m$, we get

$$
\sum_{i=1}^{m} d\left(e_{i}\right)^{2} \sum_{i=1}^{m} 1-\left(\sum_{i=1}^{m} d\left(e_{i}\right)\right)^{2} \geq \sum_{i=1}^{m} d\left(e_{i}\right) \sum_{i=1}^{m} \frac{1}{d\left(e_{i}\right)}-\left(\sum_{i=1}^{m} 1\right)^{2}
$$

expanding, we have

$$
\begin{aligned}
& \left(\begin{array}{l}
M_{1}^{3}(G)+M_{2}^{1}(G) \\
+4 m-4 M_{1}^{2}(G)
\end{array}\right) m-\left(M_{1}^{2}(G)-2 m\right)^{2} \\
& \geq\left(M_{1}^{2}(G)-2 m\right) \operatorname{IED}(G)-m^{2},
\end{aligned}
$$

and the equality holds if and only if $d(u)+d(v)$ is a constant for any edge $u v$, which completes our claim.

Corollary 3.11. With the assumptions in Theorem 3.10, we have

$$
\begin{aligned}
E M_{1}^{2}(G) \geq & \frac{M_{1}^{2}(G)^{2}}{m}+\frac{1}{m}\left[M_{1}^{2}(G)-2 m\right] \operatorname{IED}(G) \\
& -4 M_{1}^{2}(G)+3 m
\end{aligned}
$$

equality holds if and only if $d(u)+d(v)$ is a constant for any edge uv.

Remark 3.12. Still the lower bound in (3.10) and (3.13) are incomparable. Consider the Helm $H_{n}(n \geq 4)$, for which the lower bound in (3.10) is finer than (3.13) and for the complement $H_{n}(n \geq 4)$ the lower bound in (3.13) is finer than (3.10).

Our next interest is to reduce the deviation for the lower bounds from the first reformulated Zagreb index. To achieve our goal, either we need to fit some good lower bounds for $M_{1}^{3}$ or some good upper bounds for $M_{1}^{2}(G)$ in the equality (2.1).

In 2011, Ilić and Liu [10] proposed a new upper bound for the first Zagreb index $M_{1}^{2}(G)$ and proved that it has the smallest deviation from the first Zagreb index. 
Lemma 3.13. Let $G$ be a simple regular graph with $n$ vertices and $m$ edges, with a vertices of maximum degree $\Delta$ and $b$ vertices of minimum degree $\delta$. Then

$M_{1}^{2}(G) \leq 2 m(\Delta+\delta)-n \Delta \delta-(\Delta-\delta-1)(n-a-b)$,

equality holds if and only if the vertex degrees are equal to $\delta, \delta+1, \Delta-1$ or $\Delta$.

Thus we can state the following result.

Corollary 3.14. With the assumptions in Lemma 3.13, we have

$$
\begin{aligned}
E M_{1}^{2}(G) \geq & M_{1}^{3}(G)+2 M_{2}^{1}(G)-8 m(\Delta+\delta)+4 n \Delta \delta \\
& +4(\Delta-\delta-1)(n-a-b)+4 m,
\end{aligned}
$$

To the best knowledge of the authors, still now in the literature (3.14) is superior upper bound for the first Zagreb index $M_{1}^{2}(G)$. This motivates us to present the new upper bound which is stronger than (3.14) and which leads to be the better lower bound for the first reformulated Zagreb index $\operatorname{EM}_{1}^{2}(G)$.

Theorem 3.15. Let $G$ be a simple graph with $n$ nonisolated vertices and $m$ edges, with a vertices of maximum degree $\Delta$ and $b$ vertices of minimum degree $\delta$. Then

$$
\begin{aligned}
M_{1}^{2}(G) \leq & a \Delta^{2}+b \delta^{2}+(2 m-a \Delta-b \delta)(\Delta+\delta+1) \\
& +(\Delta-1)(\delta+1)\left[I D(G)-\frac{a}{\Delta}-\frac{b}{\delta}\right] \\
& -(\Delta \delta-2 \Delta-1)(n-a-b),
\end{aligned}
$$

equality holds if and only if the vertex degrees are equal to $\delta, \delta+1, \Delta-1$ or $\Delta$.

Proof. Let $a, A \in \mathbb{R}$ and $x_{i}, y_{i}$ be two sequences with the property $a y_{i} \leq x_{i} \leq A y_{i}$ for $i=1,2, \ldots, n$ and $w_{i}$ be any sequence of positive real numbers, it holds

$$
w_{i}\left(A y_{i}-x_{i}\right)\left(x_{i}-a y_{i}\right) \geq 0 \text {. }
$$

Since $w_{i}$ is a positive sequence, choose $w_{i}=m_{i}-n_{i}$ such that $m_{i} \geq n_{i}$ and by setting $A=\Delta, \quad a=\delta$, $x_{i}=d\left(v_{i}\right), \quad y_{i}=1, \quad m_{i}=1$ and $n_{i}=\frac{1}{d\left(v_{i}\right)}$ and adding over the vertices by restricting $\delta<d\left(v_{i}\right)<\Delta$, we have

$$
\begin{aligned}
& \sum_{\delta<d\left(v_{i}\right)<\Delta}\left(1-\frac{1}{d\left(v_{i}\right)}\right)\left(\Delta-d\left(v_{i}\right)\right)\left(d\left(v_{i}\right)-\delta\right) \\
\geq & \sum_{\delta<d\left(v_{i}\right)<\Delta}\left(1-\frac{1}{d\left(v_{i}\right)}\right)(\Delta-\delta-1) \geq 0,
\end{aligned}
$$

\begin{tabular}{|c|c|c|c|c|c|c|c|c|}
\hline \multirow[b]{2}{*}{$\mathrm{n}$} & \multicolumn{2}{|c|}{ Parameters } & \multicolumn{3}{|c|}{ Parameters } & \multicolumn{3}{|c|}{ Lemma 3.13} \\
\hline & Count & Avg. & Avg. & Stdev. & Eq. & Avg. & Stdev. & Eq. \\
\hline 3 & 2 & 9.0000 & 9.0000 & 0.0000 & 2 & 9.0000 & 0.0000 & 2 \\
\hline 4 & 6 & 19.6667 & 19.6667 & 0.0000 & 6 & 19.6667 & 0.0000 & 6 \\
\hline 5 & 21 & 35.4286 & 35.4286 & 0.0000 & 21 & 35.4286 & 0.0000 & 21 \\
\hline 6 & 112 & 55.6607 & 55.7440 & 0.3883 & 106 & 55.7857 & 0.5825 & 106 \\
\hline 7 & 853 & 82.6260 & 83.0240 & 1.1093 & 683 & 83.1981 & 1.5869 & 683 \\
\hline 8 & 11117 & 118.4507 & 119.6036 & 2.4343 & 6658 & 120.0452 & 3.3453 & 6658 \\
\hline 9 & 261080 & 166.1056 & 168.6350 & 4.5110 & 105659 & 169.4830 & 5.9966 & 105659 \\
\hline
\end{tabular}

expanding the above inequality gives the required result with equality if and only if $d\left(v_{i}\right)=\delta+1$ or

\begin{tabular}{|c|c|c|c|c|c|c|c|c|}
\hline \multirow[b]{2}{*}{$\mathrm{n}$} & \multicolumn{2}{|c|}{ Parameters } & \multicolumn{3}{|c|}{ Parameters } & \multicolumn{3}{|c|}{ Lemma 3.13} \\
\hline & Count & Avg. & Avg. & Stdev. & Eq. & Avg. & Stdev. & Eq. \\
\hline 10 & 106 & 44.5849 & 44.7311 & 0.4200 & 91 & 44.8019 & 0.6219 & 91 \\
\hline 11 & 235 & 50.0255 & 50.2645 & 0.6064 & 189 & 50.3787 & 0.8921 & 189 \\
\hline 12 & 551 & 55.4011 & 55.7551 & 0.8162 & 413 & 55.9220 & 1.1936 & 413 \\
\hline 13 & 1301 & 60.7640 & 61.2498 & 1.0509 & 913 & 61.4766 & 1.5299 & 913 \\
\hline 14 & 3159 & 66.1292 & 66.7626 & 1.3027 & 2075 & 67.0560 & 1.8898 & 2075 \\
\hline 15 & 7741 & 71.4949 & 72.2915 & 1.5748 & 4774 & 72.6583 & 2.2783 & 4774 \\
\hline 16 & 19320 & 76.8598 & 77.8323 & 1.8611 & 11214 & 78.2780 & 2.6862 & 11214 \\
\hline 17 & 48629 & 82.2303 & 83.3931 & 2.1644 & 26619 & 83.9238 & 3.1180 & 26619 \\
\hline 18 & 123867 & 87.6032 & 88.9683 & 2.4803 & 64057 & 89.5893 & 3.5673 & 64057 \\
\hline 19 & 317955 & 92.9794 & 94.5599 & 2.8104 & 155575 & 95.2768 & 4.0365 & 155575 \\
\hline 20 & 823065 & 98.3585 & 100.1660 & 3.1522 & 381521 & 100.9836 & 4.5219 & 381521 \\
\hline
\end{tabular}
$d\left(v_{i}\right)=\Delta-1$. This completes the proof.

The computational results for connected graphs on $n=$ 3 to 9 vertices and connected trees on $n=10$ to 20 vertices are provided in Table 1 and Table 2 respectively.

Table 1. Upper bound comparision of $M_{1}^{2}(G)$ for small graphs

Table 2. Upper bound comparison of $M_{1}^{2}(G)$ for small trees

In the group one of the Table 1 and Table 2, the first vertices and the third one has the average value of the first column represents the degree of the vertex $n$, the second column contains number of connected graphs (trees) on $n$ 
represent the average value of the upper bound, the standard deviation

$$
\left(\sqrt{\frac{\sum_{G}\left(X(G)-M_{1}^{2}(G)\right)^{2}}{\text { count }}}\right)
$$

and the number of graphs with equality case.

On comparison of the computational results in Table 1 and Table 2, we observe it is coherent that the upper bound (3.16) has the least deviation from $M_{1}^{2}(G)$ and stronger than (3.14).

Corollary 3.16. With the assumptions in Theorem 3.15, we have

$$
\begin{aligned}
E M_{1}^{2}(G) \geq & M_{1}^{3}(G)+2 M_{2}^{1}(G) \\
& +4(\Delta-\delta-1)(n-a-b)+4 m \\
& -4(\Delta-1)(\delta+1)\left[I D(G)-\frac{a}{\Delta}-\frac{b}{\delta}\right](3.18) \\
& -4\left(a \Delta^{2}+b \delta^{2}\right) \\
& -4(2 m-a \Delta-b \delta)(\Delta+\delta+1)
\end{aligned}
$$

equality holds if and only if the vertex degrees are equal

\begin{tabular}{|c|c|c|c|c|c|c|c|c|}
\hline \multicolumn{3}{|c|}{ Parameters } & \multicolumn{3}{|c|}{ Corollary 3.16} & \multicolumn{3}{|c|}{ Corollary 3.14} \\
\hline $\mathrm{n}$ & Count & Avg. & Avg. & Stdev. & Eq. & Avg. & Stdev. & Eq. \\
\hline 3 & 2 & 7.0000 & 7.0000 & 0.0000 & 2 & 7.0000 & 0.0000 & 2 \\
\hline 4 & 6 & 34.6667 & 34.6667 & 0.0000 & 6 & 34.6667 & 0.0000 & 6 \\
\hline 5 & 21 & 96.2857 & 96.2857 & 0.0000 & 21 & 96.2857 & 0.0000 & 21 \\
\hline 6 & 112 & 195.9643 & 195.6310 & 1.5533 & 106 & 195.4643 & 2.3299 & 106 \\
\hline 7 & 853 & 356.3329 & 354.7409 & 4.4371 & 683 & 354.0445 & 6.3475 & 683 \\
\hline 8 & 11117 & 605.4936 & 600.8817 & 9.7374 & 6658 & 599.1156 & 13.3811 & 6658 \\
\hline \multirow[t]{2}{*}{9} & 261080 & 991.0099 & 980.8922 & 18.0439 & 105659 & 977.5002 & 23.9863 & 105659 \\
\hline & \multicolumn{2}{|c|}{ Parameters } & \multicolumn{3}{|c|}{ Theorem 3.7} & \multicolumn{3}{|c|}{ Corollary 3.11} \\
\hline $\mathrm{n}$ & Count & Avg. & Avg. & Stdev. & Eq. & Avg. & Stdev. & Eq. \\
\hline 3 & 2 & 7.0000 & 6.7500 & 0.3536 & 1 & 7.0000 & 0.0000 & 2 \\
\hline 4 & 6 & 34.6667 & 33.1389 & 2.1093 & 2 & 34.3500 & 0.4732 & 3 \\
\hline 5 & 21 & 96.2857 & 91.2316 & 6.4066 & 2 & 94.3278 & 2.3736 & 4 \\
\hline 6 & 112 & 195.9643 & 185.4258 & 13.0763 & 5 & 190.4460 & 6.3778 & 7 \\
\hline 7 & 853 & 356.3329 & 336.8463 & 23.2882 & 4 & 344.2109 & 13.6459 & 7 \\
\hline 8 & 11117 & 605.4936 & 573.8401 & 36.7729 & 17 & 583.5165 & 24.4070 & 20 \\
\hline 9 & 261080 & 991.0099 & 942.5787 & 54.9013 & 22 & 954.9776 & 39.5418 & 27 \\
\hline
\end{tabular}
to $\delta, \delta+1, \Delta-1$ or $\Delta$.

In analogy, Table 3 - Table 4 conclude that the lower

\begin{tabular}{|c|c|c|c|c|c|c|c|c|}
\hline \multicolumn{3}{|c|}{ Parameters } & \multicolumn{3}{|c|}{ Corollary 3.16} & \multicolumn{3}{|c|}{ Corollary 3.14} \\
\hline$n$ & Count & Avg. & Avg. & Stdev. & Eq. & Avg. & Stdev. & Eq. \\
\hline 10 & 106 & 99.4151 & 98.8302 & 1.6798 & 91 & 98.5472 & 2.4877 & 91 \\
\hline 11 & 235 & 114.4340 & 113.4780 & 2.4256 & 189 & 113.0213 & 3.5682 & 189 \\
\hline 12 & 551 & 128.7477 & 127.3316 & 3.2649 & 413 & 126.6642 & 4.7744 & 413 \\
\hline 13 & 1301 & 142.8932 & 140.9500 & 4.2035 & 913 & 140.0430 & 6.1195 & 913 \\
\hline 14 & 3159 & 156.9839 & 154.4501 & 5.2108 & 2075 & 153.2764 & 7.5592 & 2075 \\
\hline 15 & 7741 & 171.0559 & 167.8697 & 6.2993 & 4774 & 166.4023 & 9.1131 & 4774 \\
\hline 16 & 19320 & 185.1062 & 181.2163 & 7.4443 & 11214 & 179.4335 & 10.7449 & 11214 \\
\hline 17 & 48629 & 199.2024 & 194.5513 & 8.6574 & 26619 & 192.4283 & 12.4720 & 26619 \\
\hline 18 & 123867 & 213.3222 & 207.8617 & 9.9211 & 64057 & 205.3778 & 14.2694 & 64057 \\
\hline 19 & 317955 & 227.4755 & 221.1534 & 11.2414 & 155575 & 218.2861 & 16.1458 & 155575 \\
\hline 20 & 823065 & 241.6596 & 234.4294 & 12.6088 & 381521 & 231.1590 & 18.0876 & 381521 \\
\hline \multicolumn{3}{|c|}{ Parameters } & \multicolumn{3}{|c|}{ Theorem 3.7} & \multicolumn{3}{|c|}{ Corollary 3.11} \\
\hline$n$ & Count & Avg. & Avg. & Stdev. & Eq. & Avg. & Stdev. & Eq. \\
\hline 10 & 106 & 99.4151 & 71.2432 & 45.7847 & 0 & 88.7336 & 13.3439 & 1 \\
\hline 11 & 235 & 114.4340 & 82.1421 & 52.2828 & 0 & 101.0084 & 17.0481 & 1 \\
\hline 12 & 551 & 128.7477 & 92.8494 & 57.5453 & 0 & 112.6745 & 20.6571 & 1 \\
\hline 13 & 1301 & 142.8932 & 103.3729 & 62.4065 & 0 & 124.1590 & 24.3165 & 1 \\
\hline 14 & 3159 & 156.9839 & 113.9656 & 66.7328 & 0 & 135.6441 & 27.8509 & 1 \\
\hline 15 & 7741 & 171.0559 & 124.5123 & 70.8548 & 0 & 147.1143 & 31.3411 & 1 \\
\hline 16 & 19320 & 185.1062 & 135.0994 & 74.7407 & 0 & 158.6074 & 34.7026 & 1 \\
\hline 17 & 48629 & 199.2024 & 145.7020 & 78.5596 & 0 & 170.1444 & 38.0023 & 1 \\
\hline 18 & 123867 & 213.3222 & 156.3424 & 82.3052 & 0 & 181.7243 & 41.2101 & 1 \\
\hline 19 & 317955 & 227.4755 & 167.0016 & 86.0265 & 0 & 193.3383 & 44.3579 & 1 \\
\hline 20 & 823065 & 241.6596 & 177.6906 & 89.7288 & 0 & 204.9893 & 47.4428 & 1 \\
\hline
\end{tabular}
bound (3.18) has the least deviation from $M_{1}^{2}(G)$ and so it is superior than the existing lower bounds.

Table 3. Lower bound comparison of $E M_{1}^{2}(G)$ for small graphs

Table 4. Lower bound comparison of $E M_{1}^{2}(G)$ for small trees 


\section{Upper Bounds on the First Reformulated Zagreb Index}

In 2010, Zhou and Trinajstić [20] proposed the first upper bound for $E M_{1}^{2}(G)$.

Lemma 4.1. Let $G$ be a graph on $n$ vertices and $m \geq 1$ edges. Then

$$
E M_{1}^{2}(G) \leq(n-4) M_{1}^{2}(G)+4 M_{2}^{1}(G)-4 m^{2}+4 m(4.1)
$$

equality holds if and only if any two non-adjacent vertices have equal degrees.

In 2012, Ilić and Zhou [11] obtained the following upper bound for $E M_{1}^{2}(G)$.

Lemma 4.2. Let $G$ be a simple graph with $n$ vertices and medges. Then

$$
\begin{aligned}
E M_{1}^{2}(G) \leq & (\Delta+\delta-4) M_{1}^{2}(G) \\
& +2 M_{2}^{1}(G)-2 m \Delta \delta+4 m
\end{aligned}
$$

equality holds if and only if $G$ is a regular or bidegreed graph.

In the same time period, De [6] Proposed the following upper bounds for $E M_{1}^{2}(G)$.

Lemma 4.3. Let $G$ be a simple graph with $n$ vertices and $m$ edges. Then

$$
\begin{gathered}
E M_{1}^{2}(G) \leq 2(\Delta+\delta-2) M_{1}^{2}(G)-4 m(\Delta \delta-1) \\
E M_{1}^{2}(G) \leq\left(M_{1}^{2}(G)-2 m\right)(m+2 \delta-3) \\
-2 m(m-1)(\delta-1)
\end{gathered}
$$

equality holds if and only if $G$ is regular.

In 2016, Milovanović et. al. [16] obtained some new upper bounds for the first reformulated Zagreb index.

Lemma 4.4. Let $G$ be a simple graph with $n(n \geq 2)$ vertices and $m$ edges. Then

$$
\begin{aligned}
E M_{1}^{2}(G) \leq & \frac{M_{1}^{2}(G)^{2}}{2 m}+2 M_{2}^{1}(G) \\
& -4 M_{1}^{2}(G)+\frac{m}{2}(\Delta-\delta)^{2}+4 m
\end{aligned}
$$

equality holds if and only if $G$ is regular or $G \cong K_{1, n-1}$. Moreover,

$$
\begin{gathered}
E M_{1}^{2}(G) \leq \frac{\left(M_{1}^{2}(G)-2 m\right)^{2}}{m}+m(\Delta-\delta)^{2} \\
E M_{1}^{2}(G) \leq \frac{\left(M_{1}^{2}(G)-2 m\right)^{2}}{m}+2 m\left(M_{1}^{2}(G)-\frac{4 m^{2}}{n}\right)
\end{gathered}
$$

equality holds if and only if $G$ is regular.

Remark 4.5. The upper bounds in (4.2) and (4.3) are emerged from Diaz-metcalf inequality, by considering the vertex and edge degrees respectively. At first, it is easy to see that the bounds in (4.1) and (4.2) are always better than (4.3) and (4.4). Secondly, the upper bound (4.2) is always better than the upper bounds (4.5), (4.6) and (4.7), so we leave it to the fascinated reader. In addition, the bounds in (4.1) and (4.2) are incomparable. For Helm Graphs $H_{n}$, (4.2) is better than (4.1), and for the Flower graphs $F_{n}$, (4.1) is better than (4.2).

Next, we are ready to present our inequality that determines upper bound for the forgotten topological index $M_{1}^{3}(G)$ in terms of $n, m, \Delta, M_{1}^{2}(G)$, and $M_{2}^{1}(G)$, which leads to the upper bound for $\operatorname{EM}_{1}^{2}(G)$.

Theorem 4.6. Let $G$ be a simple graph with $n$ nonisolated vertices and $m$ edges, with a vertices of maximum degree $\Delta$ and $b$ vertices of minimum degree $\delta$. Then

$$
\begin{aligned}
M_{1}^{3}(G) \leq & a \Delta^{3}+b \delta^{3}+(\Delta+\delta)\left(\begin{array}{c}
M_{1}^{2}(G)-a\left(\Delta^{2}+1\right) \\
-b\left(\delta^{2}+1\right)-n
\end{array}\right) \\
& +(\Delta-1)(\delta+1)\left[I D(G)-\frac{a}{\Delta}-\frac{b}{\delta}\right] \\
& -((\Delta-1)(\delta+1)-1)(2 m-a \Delta-b \delta)
\end{aligned}
$$

equality holds if and only if the vertex degrees are equal to $\delta, \delta+1, \Delta-1$ or $\Delta$.

Proof. The proof follows the same terminology of Theorem 3.15 , by the choice of setting $m_{i}=d\left(v_{i}\right)$ and $n_{i}=\frac{1}{d\left(v_{i}\right)}$.

Corollary 4.7. With the assumptions in Theorem 4.6, we have

$$
\begin{aligned}
& E M_{1}^{2}(G) \\
& \leq a \Delta^{3}+b \delta^{3}+(\Delta-1)(\delta+1)\left[I D(G)-\frac{a}{\Delta}-\frac{b}{\delta}\right] \\
& +(\Delta-\delta)\left(M_{1}^{2}(G)-a\left(\Delta^{2}+1\right)-b\left(\delta^{2}+1\right)-n\right) \\
& +2 M_{2}^{1}(G)-((\Delta-1)(\delta+1)-1)(2 m-a \Delta-b \delta) \\
& -4 M_{1}^{2}(G)+4 m
\end{aligned}
$$

equality holds if and only if the vertex degrees are equal to $\delta, \delta+1, \Delta-1$ or $\Delta$.

Corollary 4.8. Let $G$ be a simple graph with $n$ vertices and $m$ edges, with a vertices of maximum degree $\Delta$ and $b$ vertices of minimum degree $\delta$. Then

$$
\begin{aligned}
& E M_{1}^{2}(G) \\
& \leq a \Delta^{3}+b \delta^{3}+(\Delta+\delta+1)\left(M_{1}^{2}(G)-a \Delta^{2}-b \delta^{2}\right) \\
& +(\Delta-1)(\delta+1)(n-a-b) \\
& -(\Delta \delta+2 \Delta-1)(2 m-a \Delta-b \delta) \\
& +2 M_{2}^{1}(G)-4 M_{1}^{2}(G)+4 m
\end{aligned}
$$

equality holds if and only if the vertex degrees are equal to $\delta, \delta+1, \Delta-1$ or $\Delta$.

Proof. The proof follows the same terminology of Theorem 3.15, by the choice of setting $m_{i}=d\left(v_{i}\right)$ and $n_{i}=1$ and using the equality (2.1). 
From Table 5-Table 6 (with same notations in Table 1Table 2), our upper bound (4.10) has the small deviation from $M_{1}^{2}(G)$ which concludes that (4.10) is the superior than the existing upper bounds.

We end the paper by remarking that the lower and upper bounds of $\operatorname{EM}_{1}^{2}(G)$ is determined in terms of $n$; $m$; $I D(G), M_{1}^{2}(G)$ and $M_{2}^{1}(G)$. In addition, we presented the upper bound for the first Zagreb index $M_{1}^{2}(G)$ and the forgotten topological index $M_{1}^{3}(G)$ and with the assistance of computational results, we conclude that these inequalities are superior than the other bounds appeared in the literature so far.

Table 5. Upper bound comparison of $E M_{1}^{2}(G)$ for small graphs

\begin{tabular}{|c|c|c|c|c|c|c|c|c|}
\hline \multicolumn{3}{|c|}{ Parameters } & \multicolumn{3}{|c|}{ Corollary 4.8} & \multicolumn{3}{|c|}{ Corollary 4.7} \\
\hline$n$ & Count & Avg. & Avg. & Stdev. & Eq. & Avg. & Stdev. & Eq. \\
\hline 3 & 2 & 7.0000 & 7.0000 & 0.0000 & 2 & 7.0000 & 0.0000 & 2 \\
\hline 4 & 6 & 34.6667 & 34.6667 & 0.0000 & 6 & 34.6667 & 0.0000 & 6 \\
\hline 5 & 21 & 96.2857 & 96.2857 & 0.0000 & 21 & 96.2857 & 0.0000 & 21 \\
\hline 6 & 112 & 195.9643 & 196.2143 & 1.1650 & 106 & 196.2976 & 1.5533 & 106 \\
\hline 7 & 853 & 356.3329 & 357.6764 & 3.8272 & 683 & 358.0744 & 4.9305 & 683 \\
\hline 8 & 11117 & 605.4936 & 609.8366 & 9.4456 & 6658 & 610.9895 & 11.8628 & 6658 \\
\hline 9 & 261080 & 991.0099 & 1001.5845 & 19.3717 & 105659 & 1004.1139 & 23.8496 & 105659 \\
\hline & \multicolumn{2}{|c|}{ Parameters } & \multicolumn{3}{|c|}{ Lemma 4.1} & \multicolumn{3}{|c|}{ Lemma 4.2} \\
\hline$n$ & Count & Avg. & Avg. & Stdev. & Eq. & Avg. & Stdev. & Eq. \\
\hline 3 & 2 & 7.0000 & 7.0000 & 0.0000 & 2 & 7.0000 & 0.0000 & 2 \\
\hline 4 & 6 & 34.6667 & 35.3333 & 1.6330 & 5 & 35.3333 & 1.1547 & 4 \\
\hline 5 & 21 & 96.2857 & 99.6190 & 5.9362 & 11 & 99.6190 & 4.9570 & 8 \\
\hline 6 & 112 & 195.9643 & 205.9107 & 15.0772 & 36 & 206.0000 & 13.0767 & 15 \\
\hline 7 & 853 & 356.3329 & 380.2673 & 31.8992 & 91 & 378.1290 & 26.5012 & 25 \\
\hline 8 & 11117 & 605.4936 & 652.0770 & 58.6369 & 471 & 644.4005 & 45.5048 & 53 \\
\hline 9 & 261080 & 991.0099 & 1072.0948 & 98.1035 & 2296 & 1052.3611 & 70.0854 & 95 \\
\hline
\end{tabular}

Table 6. Upper bound comparison of $E M_{1}^{2}(G)$ for small trees

\begin{tabular}{|c|c|c|c|c|c|c|c|c|}
\hline \multicolumn{3}{|c|}{ Parameters } & \multicolumn{3}{|c|}{$\begin{array}{l}\text { Corollary } 4.8 \\
\end{array}$} & \multicolumn{3}{|c|}{ Corollary 4.7} \\
\hline$n$ & Count & Avg. & Avg. & Stdev. & Eq. & Avg. & Stdev. & Eq. \\
\hline 10 & 106 & 99.4151 & 99.8679 & 1.3175 & 91 & 100.0142 & 1.7358 & 91 \\
\hline 11 & 235 & 114.4340 & 115.1830 & 1.9439 & 189 & 115.4220 & 2.5469 & 189 \\
\hline 12 & 551 & 128.7477 & 129.8730 & 2.6727 & 413 & 130.2270 & 3.4831 & 413 \\
\hline 13 & 1301 & 142.8932 & 144.4520 & 3.4937 & 913 & 144.9377 & 4.5362 & 913 \\
\hline 14 & 3159 & 156.9839 & 159.0326 & 4.3828 & 2075 & 159.6660 & 5.6745 & 2075 \\
\hline 15 & 7741 & 171.0559 & 173.6478 & 5.3461 & 4774 & 174.4444 & 6.9074 & 4774 \\
\hline 16 & 19320 & 185.1062 & 188.2864 & 6.3635 & 11214 & 189.2589 & 8.2089 & 11214 \\
\hline 17 & 48629 & 199.2024 & 203.0205 & 7.4434 & 26619 & 204.1833 & 9.5900 & 26619 \\
\hline 18 & 123867 & 213.3222 & 217.8203 & 8.5705 & 64057 & 219.1854 & 11.0310 & 64057 \\
\hline 19 & 317955 & 227.4755 & 232.6989 & 9.7500 & 155575 & 234.2795 & 12.5388 & 155575 \\
\hline \multirow[t]{2}{*}{20} & 823065 & 241.6596 & 247.6490 & 10.9734 & 381521 & 249.4566 & 14.1024 & 381521 \\
\hline & \multicolumn{2}{|c|}{ Parameters } & \multicolumn{3}{|c|}{ Lemma 4.1} & \multicolumn{3}{|c|}{ Lemma 4.2} \\
\hline$n$ & Count & Avg. & Avg. & Stdev. & Eq. & Avg. & Stdev. & Eq. \\
\hline 10 & 106 & 99.4151 & 113.8491 & 16.2724 & 5 & 164.3774 & 68.8660 & 1 \\
\hline 11 & 235 & 114.4340 & 133.5319 & 21.2783 & 3 & 201.2426 & 91.8521 & 1 \\
\hline 12 & 551 & 128.7477 & 152.3194 & 26.3422 & 5 & 240.4646 & 118.3697 & 1 \\
\hline 13 & 1301 & 142.8932 & 171.5634 & 31.9734 & 2 & 282.1168 & 147.5565 & 1 \\
\hline 14 & 3159 & 156.9839 & 190.7692 & 37.7317 & 10 & 326.6477 & 179.9576 & 1 \\
\hline 15 & 7741 & 171.0559 & 210.3741 & 43.8713 & 2 & 373.8465 & 215.0847 & 1 \\
\hline 16 & 19320 & 185.1062 & 230.0205 & 50.1354 & 9 & 423.8166 & 253.1651 & 1 \\
\hline 17 & 48629 & 199.2024 & 249.9992 & 56.6729 & 6 & 476.5955 & 294.0235 & 1 \\
\hline 18 & 123867 & 213.3222 & 270.1175 & 63.3533 & 16 & 532.1868 & 337.7529 & 1 \\
\hline 19 & 317955 & 227.4755 & 290.4733 & 70.2383 & 2 & 590.5895 & 384.2730 & 1 \\
\hline 20 & 823065 & 241.6596 & 310.9927 & 77.2696 & 27 & 651.8210 & 433.6323 & 1 \\
\hline
\end{tabular}

\section{References}

[1] M. Ali Rostami, H. Martin B ucker and A. Azadi, Illustrating a Graph Coloring Algorithm Based on the Principle of Inclusion and Exclusion Using GraphTea, LNCS, Springer. 8719 (2014) 514-517.
[2] A.R. Ashrafi, T. Došlić and A.Hamzeha, The Zagreb coindices of graph operations, Discrete Appl. Math. 158 (2010) 1571-1578.

[3] M. Bianchi, A. Cornaro, J. L. Palacios, A. Torriero, New bounds of degree-based topological indices for some classes of c-cyclic graphs. Discrete App. Math. 184 (2015) 62-75.

[4] G.B.A. Xavier, E. Suresh and I. Gutman, Counting relations for general Zagreb indices, Kragujevac J. Math. 38 (2014) 95-103. 
[5] K. C. Das, K. Xu and J. Nam, Zagreb indices of graphs, Front. Math. China 10 (2015) 567-582.

[6] N. De, Some bounds of reformulated Zagreb indices, Appl. Math. Sci. 6 (2012) 5005-5012.

[7] S. Fajtlowicz S, On conjectures of graffiti II, Congr. Numer. 60 (1987), 189-197.

[8] B. Fortula and I. Gutman, A forgotten topological index, J. Math. Chem. 53 (2015) 1184-1190.

[9] I. Gutman, B. Ruščić, N. Trinajstić and C. F. Wilcox, Graph theory and molecular orbitals, XII. Acyclic polyenes, J. Chem. Phys. 62 (1975) 3399-3405.

[10] A. Ilić, M. Ilić and B. Liu, On the upper bounds for the first Zagreb index, Kragujevac Journal of Math. 35 (2011) 173-182.

[11] A. Ilić and B. Zhou, On reformulated Zagreb indices, Discrete App. Math. 160 (2012) 204-209.

[12] S. Ji, X. Li and B. Huo, On Reformulated Zagreb Indices with Respect to Acyclic, Unicyclic and Bicyclic Graphs, MATCH Commun. Math. Comput. Chem. 72 (2014) $723\{732$.
[13] X. Li and J. Zheng, A unified approach to the extremal trees for different indices, MATCH Commun. Math. Comput. Chem. 54 (2005) 195-208.

[14] T. Mansour and C. Song, The $a$ and $(a, b)-$ Analogs of Zagreb Indices and Coindices of Graphs, Intern. J. Combin. (2012) ID 909285.

[15] A. Miličević, S. Nikolić and N. Trinajstić, On reformulated Zagreb indices, Mol. Divers. 8 (2004) 393-399.

[16] E.I. Milovanović, I.Ž. Milovanović, E.Ć. Dolićanin and E. Glogić, A note on the first reformulated Zagreb index, Appl. Math. Comp. 273 (2016) 16-20.

[17] G. Su, L. Xiong, L. Xu and B. Ma, On the maximum and minimum first reformulated Zagreb index with connectivity bat most $k$, FILMAT 25 (2011) 75-83.

[18] K. Xu and K.C. Das, Some extremal graphs with respect to inverse degree, Discrete App. Math. (2015).

[19] B. Zhou and N. Trinajstić, On general sum-connectivity index, $J$. Math. Chem. 47 (2010) 210-218.

[20] B. Zhou and N. Trinajstić, Some properties of the reformulated Zagreb indices, J. Math. Chem. 48 (2010) 714-719. 